\title{
Refractory Rectal Adenocarcinoma
}

National Cancer Institute

\section{Source}

National Cancer Institute. Refractory Rectal Adenocarcinoma. NCI Thesaurus. Code C162157.

Rectal adenocarcinoma that is resistant to treatment. 